\title{
Relativistic, model-independent, three-particle quantization condition
}

\author{
Maxwell T. Hansen \\ Physics Department, University of Washington, Seattle, WA 98195-1560, USA \\ E-mail: mth28@uw. edu
}

\section{Stephen R. Sharpe}

Physics Department, University of Washington, Seattle, WA 98195-1560, USA

E-mail: srsharpeduw.edu

\begin{abstract}
This is a combined write-up for two talks which were given consecutively and which described different aspects of the same topic. We present a generalization of Lüscher's relation between the finite-volume spectrum and $S$-matrix to three particles. Specifically, we consider a scalar field theory, which has a $\mathbb{Z}_{2}$ symmetry that prevents even/odd coupling. The theory is assumed to have no two-particle bound-states and to have a two-particle scattering phase shift that is bounded by $\pi / 2$ in the regime of elastic scattering. Considering center of mass energies between one and five particle masses, we evaluate a three-to-three finite-volume correlator to all orders in perturbation theory. Only terms which are exponentially suppressed in volume are neglected. From poles in the correlator we then determine the relation between finite-volume spectrum and scattering quantities. In this analysis one must carefully treat the unitary cusp at two-particle threshold. This point, which was neglected in the conference talks, is described in some detail here. We also describe an important check on our main result by reproducing the large volume expansion of the energy shift from the three-particle threshold. This is found to be consistent with previous work through four non-trivial orders.
\end{abstract}

The 31st International Symposium on Lattice Field Theory

July 29 - August 3, 2013

Mainz, Germany 


\section{Introduction}

Numerical lattice QCD (LQCD) is the only known systematic method for determining nonperturbative, low-energy properties of the strong interaction. However, it is only possible to determine QCD correlators defined in Euclidean time. This presents a serious challenge for extracting multi-hadron scattering amplitudes.

In the case of two-to-two scattering, the issue was resolved by Martin Lüscher in a series of papers from 1986-1991 [1-3]. His central insight was that one can use finite spatial volume as a tool to recover infinite-volume scattering information. Specifically, he derived a relation between the discrete finite-volume spectrum and the elastic scattering amplitude, valid below the inelastic threshold. This approach has since been implemented in numerous numerical studies, allowing extraction of scattering phases and determination of resonance properties.

Given the robust body of theoretical and numerical work in the two-particle sector, it is natural to investigate whether the relation between the finite-volume spectrum and scattering-amplitudes can be generalized to accommodate three (and higher) particle states. The last few years have seen important developments in this direction $[4,5]$. However, a complete method for extracting three-particle scattering amplitudes is still unavailable.

This implies, for example, that LQCD cannot yet offer predictions on the mass and width of the $\omega$ resonance, since it decays predominantly into three pions. Similarly the Roper resonance, $N(1440)$, which decays with a $40 \%$ branching fraction into three-particle states, cannot be rigorously investigated. The latter case is especially interesting since its position below $N(1535)$ contradicts quark-model predictions, with more complicated explanations so far reaching no consensus. Along similar lines, three body weak decays such as $K \rightarrow 3 \pi$ cannot yet be investigated using LQCD.

Further motivation comes from the realization that three-body forces are important for understanding strongly interacting quantum systems such as nuclei and neutron stars. Any effort to describe such systems from first-principles QCD thus requires a method for extracting threeparticle scattering from lattice simulations. As a final motivation, we recall that elastic phase shifts extracted from LQCD are currently limited to energies below inelastic threshold. Only by generalizing the formalism to include three or more particle-states can this range of validity be extended.

In this work we take a step in this direction by deriving a relation between the finite-volume spectrum of three particle states and infinite-volume two-to-two and three-to-three scattering, in the context of a relativistic scalar field theory. Here we can only sketch the derivation; details will be given in a forthcoming article [6]. We also note that some technical aspects of the result have been corrected since the talks, particularly those in section 4 .

\section{Set-up}

We assume throughout a finite, cubic spatial volume with extent $L$ and with periodic boundary conditions. We demand that $L$ is large enough to neglect exponentially suppressed corrections of the form $e^{-m L}$, where $m$ is the particle mass. Although the main target of this formalism is finite-volume lattice calculations, we assume here that discretization errors are small and have been controlled elsewhere. We therefore work throughout in continuum field theory (zero lattice 
spacing). We also differ from the standard simulation set-up by working in Minkowski rather than Euclidean time, with the time coordinate of infinite extent. Minkowski time turns out to be convenient for our analysis and the distinction is irrelevant to the final result.

We consider a scalar field theory describing particles of mass $m$. Thus, all results in this work are valid for identical particles. The Lagrangian of the theory is arbitrary except that it is invariant under a $\mathbb{Z}_{2}$ symmetry that prevents coupling between even- and odd-number particle states. (For the pion in QCD this is G-parity.) Aside from the $\mathbb{Z}_{2}$ symmetry, we make no restrictions on the Lagrangian. In particular we include all vertices with an even number of scalar fields and make no assumptions about relative coupling strengths. We further require that the theory has no twoparticle bound-states and that the two-particle phase shift above threshold does not have magnitude exceeding $\pi / 2$ below the inelastic (four-particle) threshold. These criteria are necessary to prevent poles in the two-particle K-matrix, which would invalidate our present derivation.

Following [7] we determine the spectrum from a finite-volume, Minkowski-time correlator

$$
C_{L}(E, \vec{P}) \equiv \int_{L} d^{4} x e^{i\left(-\vec{P} \cdot \vec{x}+E x^{0}\right)}\left\langle 0\left|\mathrm{~T} \sigma(x) \sigma^{\dagger}(0)\right| 0\right\rangle .
$$

Here $\mathrm{T}$ indicates time-ordering and $\sigma(x)$ is an interpolating field, with spatial periodicity $L$, that couples to states with an odd number of scalar particles. The Fourier transform restricts the states to have total energy and momentum $\left(E, \vec{P}=2 \pi \vec{n}_{P} / L\right)$, with $\vec{n}_{P}$ a vector of integers. We denote by $E^{*}$ the total energy in the center of mass $(\mathrm{CM})$ frame, $\left(E^{* 2}=E^{2}-\vec{P}^{2}\right)$. The subsequent derivation holds only when we limit the total energy to lie in the range $m<E^{*}<5 m$. This restricts the possible on-shell intermediate states to those with three particles.

At fixed $\{L, \vec{P}\}$, the spectrum of our theory is given by the set of CM energies $E_{1}^{*}, E_{2}^{*}, \cdots$ for which $C_{L}\left(\left[E^{* 2}+\vec{P}^{2}\right]^{1 / 2}, \vec{P}\right)$ has a pole. Thus our goal is to determine the poles in $C_{L}$ when we include all finite-volume contributions scaling as a power of $1 / L$. The finiteness of the volume enters our calculation through the prescription of summing (rather than integrating) the spatial components of all loop momenta:

$$
\int \frac{d^{4} k}{(2 \pi)^{4}} \longrightarrow \frac{1}{L^{3}} \sum_{\vec{k}=2 \pi \vec{n} / L} \int \frac{d k^{0}}{2 \pi} \text { with } \vec{n} \in \mathbb{Z}^{3} .
$$

We stress that when $L \rightarrow \infty$ the correlator does not have poles, in our energy range, but only cuts.

A key technical observation underlying our analysis is that finite-volume momentum sums can be replaced by integrals if the integrand is non-singular and smooth [2]. This replacement leads only to errors which are exponentially suppressed, and which we assume are negligible. This motivates organizing the sum of diagrams contributing to $C_{L}$ into the skeleton expansion shown in Fig. 1. Here we keep explicit all intermediate states which can go on shell, while collecting all off-shell contributions into Bethe-Salpeter kernels. Each diagram in the expansion contains $\tilde{\sigma}=\tilde{\sigma}\left(q^{\prime}, p^{\prime}\right)$ and $\tilde{\sigma}^{\dagger}=\tilde{\sigma}^{\dagger}(q, p)$ "endcaps" on the far left and far right respectively. The form of these functions depends on the original interpolating fields, and does not affect the final answer.

Between endcaps each diagram contains some number of two-to-two and three-to-three BetheSalpeter kernels. The two-to-two Bethe-Salpeter kernel $i B_{2 \rightarrow 2}$ is the sum of all diagrams which are two-particle irreducible in the s-channel. Given our constraint $m<E^{*}<5 m$, together with the fact that $B_{2 \rightarrow 2}$ always appears alongside a spectator line, it follows that none of the $B_{2 \rightarrow 2}$ in Fig. 1 can 


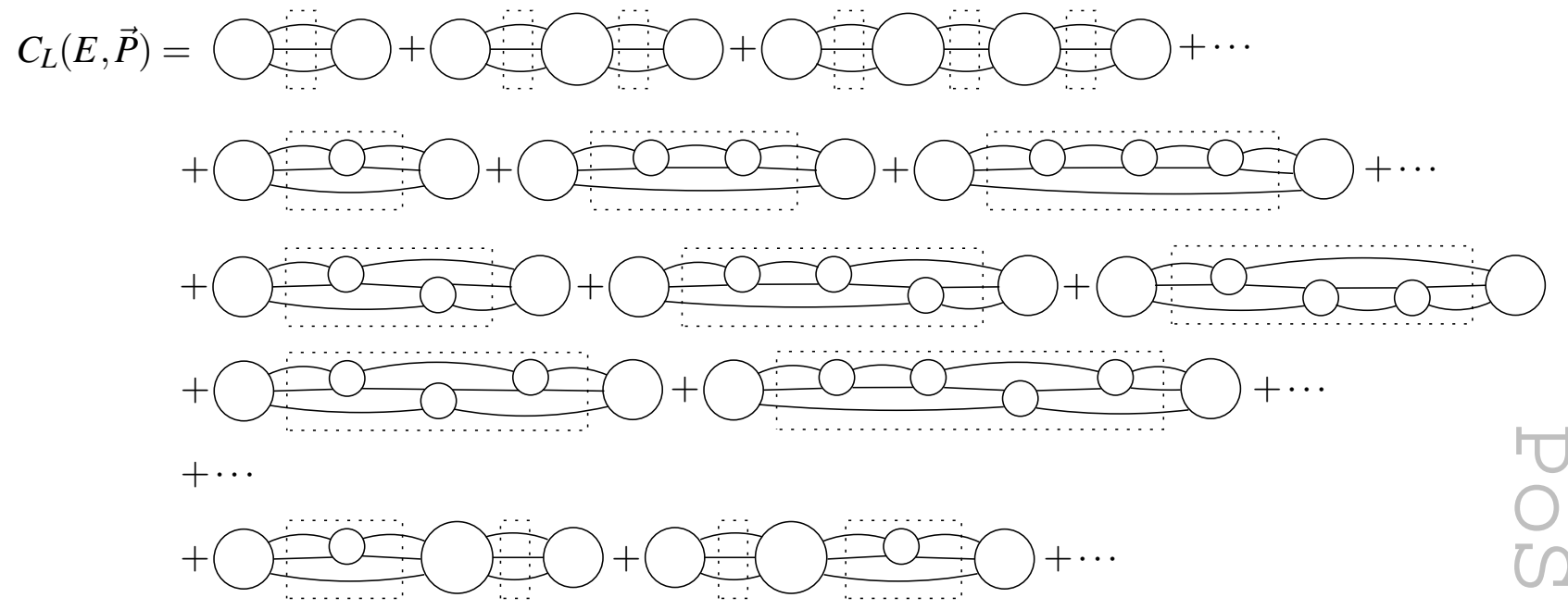

Figure 1: Skeleton expansion defining the finite-volume correlator. The leftmost circle in all diagrams represents the function $\widetilde{\sigma}$, while the rightmost represents $\widetilde{\sigma}^{\dagger}$. Any insertion between these with four (six) legs represents a two-to-two (three-to-three) Bethe-Salpeter kernel $i B_{2 \rightarrow 2}\left(i B_{3 \rightarrow 3}\right)$. All lines represent fullydressed propagators. Finally, dashed rectangles indicate that all loop momenta on enclosed propagators are summed rather than integrated. See text for further details.

have on-shell intermediate states. This implies that the integrands "inside" $B_{2 \rightarrow 2}$ are non-singular, and momentum sums can be replaced by integrals. Thus we can replace the finite-volume version of $B_{2 \rightarrow 2}$ with its infinite-volume correspondent. Similarly $i B_{3 \rightarrow 3}$ is defined so that it contains no diagram in which three propagators carry the total energy-momentum $(E, \vec{P})$. Diagrams with one propagator carrying $(E, \vec{P})$, as well as any odd number greater than three, are allowed. Again we drop exponentially suppressed corrections and work with the infinite-volume version of the kernel.

Finally, in our skeleton expansion all kernels and interpolating functions are connected by fully-dressed propagators

$$
\Delta(q) \equiv \int d^{4} x e^{i q x}\langle 0|\mathrm{~T} \phi(x) \phi(0)| 0\rangle .
$$

Here $\phi(x)$ is a one particle interpolating field defined with on-shell renormalization so that

$$
\lim _{q^{0} \rightarrow \omega_{q}}\left[\Delta(q)\left(q^{2}-m^{2}\right) / i\right]=1 .
$$

We use infinite-volume fully-dressed propagators throughout, which is justified because the selfenergy graphs do not contain on-shell intermediate states.

In summary, only three-particle intermediate states give important (power-law) finite-volume corrections. The skeleton expansion therefore keeps these on-shell states explicit and groups all off-shell states into infinite-volume kernels and propagators.

\section{3-body phase space and notation}

To avoid repetitive definitions, we describe here the coordinates and notation we use for three 
particles states, and also collect some general notation. We begin with the latter:

$$
\int_{\vec{k}} \equiv \int d^{3} k /(2 \pi)^{3}, \quad \omega_{k}=\sqrt{\vec{k}^{2}+m^{2}}, \quad \omega_{k a}=\sqrt{(\vec{P}-\vec{k}-\vec{a})^{2}+m^{2}} .
$$

For three on-shell particles having total energy-momentum $(E, \vec{P})$, we parametrize phase space by the momentum of one particle (the "spectator"), usually denoted $\vec{k}$ or $\vec{p}$, and the direction of one of the other two particles in their CM frame, usually $\hat{q}^{*}$ or $\hat{a}^{*}$. Note that the magnitude of the momentum in the two-particle CM frame is fixed by the spectator momentum. If the latter is $\vec{k}$, this magnitude is denoted by $q_{k}$, and given by

$$
\frac{q_{k}^{2}}{m^{2}}=x_{k}-1, \quad x_{k}=\frac{\left(E-\omega_{k}\right)^{2}-(\vec{P}-\vec{k})^{2}}{4 m^{2}} .
$$

Thus $x_{k}=1$ at the two-particle threshold.

In finite volume, three-particle phase space is restricted. We parametrize it by a spectator momentum which now takes on quantized values, $\vec{k} \in(2 \pi / L) \mathbb{Z}^{3}$, together with the angular momentum in the CM-frame of the other two particles, $\ell, m$. Thus three-to-three scattering amplitudes become matrices with indices $k, \ell, m$, where $k$ is a shorthand for all the quantized values of $\vec{k}$. This is the natural extension of the two-particle analysis in which the matrix indices are simply $\ell, m$.

Finally, we need notation for the case where two of the three particles are on-shell, with fourmomenta, say, $\left(\omega_{p}, \vec{p}\right)$ and $\left(\omega_{k}, \vec{k}\right)$, but the third, with four-momentum $\left(E-\omega_{p}-\omega_{k}, \vec{P}-\vec{p}-\vec{k}\right)$, is not. Treating $\vec{k}$ as the spectator, we can boost to the zero-momentum frame of the other two particles, using a boost with velocity $\vec{\beta}_{k}=-(\vec{P}-\vec{k}) /\left(E-\omega_{k}\right)$. This is possible kinematically as long as $x_{k}>0$. We then denote $\left(\omega_{p^{*}}, \vec{p}^{*}\right)$ as the four-vector obtained by such a boost acting on $\left(\omega_{p}, \vec{p}\right)$, with $p^{*}$ and $\hat{p}^{*}$ being the magnitude and direction of $\vec{p}^{*}$. Since we allow the $(\vec{P}-\vec{p}-\vec{k})-$ particle to be off-shell, $p^{*}$ is not constrained. Similar definitions hold with $\vec{k}$ and $\vec{p}$ interchanged.

\section{Cusp effects and pole prescriptions}

Our method for picking out finite-volume corrections is a generalization of that used in Ref. [7] to analyze the two-particle case. To illustrate the method, consider the simplest contribution to the skeleton expansion, the first diagram in Fig. 1. Labeling its contribution $\mathscr{V}_{L}$, and that of the corresponding infinite-volume diagram $\mathscr{V}_{\infty}$, one can show that (up to exponentially small corrections)

$$
\mathscr{V}_{L}-\mathscr{V}_{\infty}=\left[\frac{1}{L^{6}} \sum_{\vec{k}, \vec{a}}-\int_{\vec{k}, \vec{a}}\right] \frac{i \sigma(\vec{k}, \vec{a}) \sigma^{\dagger}(\vec{k}, \vec{a})}{2 \omega_{k} 2 \omega_{a} 2 \omega_{k a}\left(E-\omega_{k}-\omega_{a}-\omega_{k a}+i \varepsilon\right)} .
$$

Here $\vec{k}$ and $\vec{a}$ are the momenta flowing in the bottom and top lines, respectively. We have subtracted $\mathscr{V}_{\infty}$ because this leads to simpler expressions while not affecting the pole structure (since $\mathscr{V}_{\infty}$ only has cuts). In particular, the difference is dominated by the region of summation where the summand is singular, i.e. the region where all three-particles are on-shell. It is because of this singularity that the sum-integral difference cannot be neglected.

To proceed, it turns out to be very useful to replace the integral $\int_{k}$ in the rightmost term in Eq. (4.1) with the corresponding sum. This allows $\mathscr{V}_{L}$ to be combined with the diagrams on the 
second line of Fig. 1, all of which have a "spectator" line carrying momentum $\vec{k}$. This replacement is justified if

$$
\int_{\vec{a}} \frac{i \sigma(\vec{k}, \vec{a}) \sigma^{\dagger}(\vec{k}, \vec{a})}{2 \omega_{k} 2 \omega_{a} 2 \omega_{k a}\left(E-\omega_{k}-\omega_{a}-\omega_{k a}+i \varepsilon\right)}
$$

is a smooth function of $\vec{k}$ with characteristic width $m$. Unfortunately, although (4.2) is finite and continuous, it is not smooth, due to a unitary cusp. This cusp is simply the imaginary part from the physical cut, which turns on, with infinite derivative, when one moves from below to above threshold. The threshold occurs when $x_{k}=1$ [see Eq. (3.2) above]. This cusp problem arises because we have a third particle whose momentum we are varying - it is not present in the twoparticle analysis.

We can remove the cusp by subtracting, instead of $\mathscr{V}_{\infty}$, a different infinite-volume quantity, $\widetilde{\mathscr{V}}_{\infty}$, in which the pole prescription is changed. We are free to do this as long as $\widetilde{\mathscr{V}}_{\infty}$ does not contain poles, as is the case here. Specifically, we change from the $i \varepsilon$ prescription to a modified principal-value prescription, similar to that introduced in Ref. [4]. It is defined by

$$
\widetilde{\mathrm{PV}} \frac{1}{E-\omega_{a}-\omega_{k}-\omega_{k a}} \equiv[1-i H(\vec{k}) H(\vec{a}) \operatorname{Im}] \frac{1}{E-\omega_{a}-\omega_{k}-\omega_{k a}+i \varepsilon}
$$

Here $H(\vec{k})$ is a smooth cutoff function, whose role is to damp contributions from subthreshold momenta. From analyzing more complicated diagrams, we find that it is convenient to require ${ }^{1}$

$$
\begin{gathered}
H(\vec{k})=1 \text { if } x_{k}>1 \text { and } H(\vec{k})=0 \text { if } x_{k}<0, \\
{\left[\frac{1}{L^{3}} \sum_{\vec{k}}-\int_{\vec{k}}\right] H(\vec{k})=\mathscr{O}\left(e^{-m L}\right) .}
\end{gathered}
$$

In the region where $H(\vec{k}) H(\vec{a})=1$, corresponding to all three particles being above threshold, our prescription becomes " $1-i \mathrm{Im}=\mathrm{Re}$ ". This is the standard principal value prescription. It removes the unitary cusp, provided that we correctly analytically continue below threshold. More specifically, for subthreshold momenta, i.e. those for which which $E-\omega_{k}-\omega_{a}-\omega_{k a}<0$, no pole prescription is needed, so one might think that $H$ should be required to vanish. However, such a choice produces a cusp which leads to power-law finite-volume corrections. We therefore define $\widetilde{\mathrm{PV}}$ below threshold by analytic continuation of the above-threshold result, which avoids cusps. This is implicitly included in the definition of "Im" in Eq. (4.3). The product $H(\vec{k}) H(\vec{a})$ then provides a smooth interpolation to the naive subthreshold definition.

Putting everything together we obtain, for the first diagram,

$$
\mathscr{V}_{L}-\widetilde{\mathscr{V}}_{\infty}=\frac{1}{L^{3}} \sum_{\vec{k}}\left[\frac{1}{L^{3}} \sum_{\vec{a}}-\widetilde{\mathrm{PV}} \int_{\vec{a}}\right] \frac{i \sigma(\vec{k}, \vec{a}) \sigma^{\dagger}(\vec{k}, \vec{a})}{2 \omega_{k} 2 \omega_{a} 2 \omega_{k a}\left(E-\omega_{k}-\omega_{a}-\omega_{k a}\right)} .
$$

This is now in a form which allows us to use a generalization of the result of Ref. [7] in which "sum minus integral" acting on a pole picks out the residue of the pole (which is an on-shell quantity)

\footnotetext{
${ }^{1}$ An example which satisfies all requirements is $H(\vec{k}) \equiv J\left(x_{k}\right)$ with

$$
J(x)=0 \text { for } x<0, \quad J(x)=\exp (-(1 / x) \exp [-1 /(1-x)]) \text { for } 0<x<1, \quad J(x)=1 \text { for } 1<x
$$
}


multiplied by a kinematic function (related to Lüscher's zeta-function [2, 3]). The main changes from Ref. [7] are that the kinematic function, called $\widetilde{F}$ below, differs slightly due to the change in pole prescription, and that on-shell quantities analytically continued below threshold appear.

It is straightforward to extend this analysis to the diagrams on the second line of Figure 1, together with $\mathscr{V}_{L}$. These diagrams have any number of two-to-two Bethe-Salpeter kernels appearing on the same pair of propagators. We denote the sum of all such diagrams $C_{L}^{(1)}$ and the corresponding infinite-volume quantity $C_{\infty}^{(1)}$. We find that

$$
C_{L}^{(1)}-C_{\infty}^{(1)}=\left(\sigma+A^{\prime(1)}\right) \frac{i \widetilde{F}}{2 \omega L^{3}} \frac{1}{1+\widetilde{\mathscr{K}} \widetilde{F}}\left(\sigma^{\dagger}+A^{(1)}\right)-(2 / 3) \sigma \frac{i \widetilde{F}}{2 \omega L^{3}} \sigma^{\dagger} .
$$

This expression has the form (row vector $) \times($ matrix $) \times($ column vector $)$ in the direct product space

$$
\text { [finite volume spectator momentum } \left.\left.\vec{k} \in(2 \pi / L) \mathbb{Z}^{3}\right] \times \text { angular momentum }(\ell, m)\right] \text {, }
$$

which was introduced in Sec. 3. This means that the rows $\left(\sigma, A^{\prime(1)}\right)$ and columns $\left(\sigma^{\dagger}, A^{(1)}\right)$ have one set of indices $k, \ell, m$ where $k$ is short for $\vec{k} \in(2 \pi / L) \mathbb{Z}^{3}$ and where $\ell, m$ describe the angular momentum in the CM-frame of the non-spectator pair. Here $A^{\prime(1)}$ and $A^{(1)}$ are corrections to the endcaps $\sigma$ and $\sigma^{\dagger}$, respectively, involving insertions of $B_{2 \rightarrow 2}$. Their detailed form is irrelevant for the finite-volume spectrum. The matrices $\left[2 \omega L^{3}\right]^{-1}, \widetilde{F}$ and $\widetilde{\mathscr{K}}$ have two sets of $k, \ell, m$ indices. They are defined as

$$
\begin{aligned}
{\left[\frac{1}{2 \omega L^{3}}\right]_{k^{\prime}, \ell^{\prime}, m^{\prime} ; k, \ell, m} } & \equiv \delta_{k^{\prime}, k} \delta_{\ell^{\prime}, \ell} \delta_{m^{\prime}, m} \frac{1}{2 \omega_{k} L^{3}}, \quad \widetilde{\mathscr{K}_{k^{\prime}, \ell^{\prime}, m^{\prime} ; k, \ell, m}} \equiv \delta_{k^{\prime}, k} \widetilde{\mathscr{K}_{2} ; \ell^{\prime}, m^{\prime} ; \ell, m}(\vec{k}), \\
\widetilde{F}_{k^{\prime}, \ell^{\prime}, m^{\prime} ; k, \ell, m} & \equiv \delta_{k, k^{\prime}} \frac{1}{2}\left[\frac{1}{L^{3}} \sum_{\vec{a}}-\widetilde{\mathrm{PV}} \int_{\vec{a}}\right] \frac{4 \pi Y_{\ell^{\prime}, m^{\prime}}\left(\hat{a}^{*}\right) Y_{\ell, m}^{*}\left(\hat{a}^{*}\right) H(\vec{k}) H(\vec{a})}{2 \omega_{a} 2 \omega_{k a}\left(E-\omega_{k}-\omega_{a}-\omega_{k a}\right)}\left(a^{*} / q_{k}^{*}\right)^{\ell+\ell^{\prime}} .
\end{aligned}
$$

Here $\widetilde{\mathscr{K}_{2}}$ is the two-particle K-matrix defined with our modified PV prescription. Its argument $\vec{k}$ indicates the momentum carried by the spectator, a notation used frequently below. The quantities appearing in Eq. (4.11) are defined in Sec. 3.

Aside from the spectator, the diagrams leading to the result (4.8) are exactly those contributing to the two-particle correlator studied in Ref. [7]. Thus we expect the results to be closely related. Indeed, the matrix appearing in the first term on the r.h.s. of (4.8) has the same form as the result of Ref. [7]. The second term is present here because of a mismatch of symmetry factors. If, however, one considers a theory with two identical particles plus a third which is non-interacting (therefore non-identical), then the second term is absent. Indeed, in this alternative theory we have already summed all possible diagrams, and the divergence of the first term determines the finite-volume spectrum.

This observation provides a check on the formalism presented so far. In the alternative theory, we know the spectrum to be that of a single free particle (with any finite-volume momentum, $\vec{k}$ ) combined with that of two interacting particles in the box with combined momentum $\vec{P}-\vec{k}$. The latter spectrum is itself known from Refs. [7, 8], and is obtained from the solutions of

$$
\operatorname{det}\left[\mathscr{M}_{2}^{-1}(\vec{k})+F_{2}(\vec{k})\right]=0 .
$$


Here $i \mathscr{M}_{2}$ is the two-particle scattering amplitude, while $F_{2}$ is a finite-volume kinematic factor. Both are matrices in $\ell, m$ space. $F_{2}$ is defined like $\widetilde{F}$ [Eq. (4.11)] except that the $k$ index and $\delta_{k, k^{\prime}}$ term are absent, and the pole is regulated using the $i \varepsilon$ prescription. As above, the argument $\vec{k}$ indicates the spectator momentum, so that the total momentum flowing through the two-particle correlator is $\left(E-\omega_{k}, \vec{P}-\vec{k}\right)$.

Our expression for all diagrams with one particle unscattered [Eq. (4.8) minus the last term] gives the spectrum for a free spectator combined with that obtained from the solutions of

$$
\operatorname{det}\left[\widetilde{\mathscr{K}}_{2}^{-1}(\vec{k})+\widetilde{F}_{2}(\vec{k})\right]=0 .
$$

Here $\widetilde{F}_{2}$ is same as $\widetilde{F}$, except that the $k$ index and $\delta_{k, k^{\prime}}$ are removed. These two results for the theory with one free particle agree, because Eqs. (4.12) and (4.13) turn out to be equivalent. This is because

$$
\mathscr{M}_{2}^{-1}(\vec{k})-\widetilde{\mathscr{K}}_{2}^{-1}(\vec{k})=\widetilde{F}_{2}(\vec{k})-F_{2}(\vec{k})=-i \frac{q_{k}^{*}}{32 \pi}\left[q_{k}^{* 2}+m^{2}\right]^{-1 / 2} H(\vec{k}) \mathbf{1} .
$$

Here all quantities are matrices in $\ell, m$ space, with the r.h.s. proportional to the identity. Above the two-particle threshold, where $H(\vec{k})=1$, the r.h.s is simply the imaginary part of $\mathscr{M}_{2}^{-1}$, so that $\widetilde{\mathscr{K}}_{2}^{-1}$ is the real part, which is one way of defining the standard K-matrix.

\section{Three-particle singularities}

We next turn our attention to the finite-volume diagrams on the third line of Figure 1, and in particular the part of the diagram within the dashed rectangle. We will not state the result of summing all such diagrams here. Instead we only comment that the summation contains a factor of the form

$$
i \widetilde{\mathscr{K}}_{3 \rightarrow 3}^{2, \text { unsym }}\left(\vec{k}, \hat{a}^{\prime *} ; \vec{p}, \hat{a}^{*}\right) \equiv i \widetilde{\mathscr{K}_{2}}(\vec{k})[\widetilde{\mathrm{PV}} \Delta(P-p-k)] i \widetilde{\mathscr{K}_{2}}(\vec{p}) .
$$

Here the superscript 2 on the 1.h.s indicates that the quantity has two $\mathscr{K}_{2}$ insertions, while "unsym" indicates that it is not symmetric under the exchange of external momenta. This is our first contribution to three-to-three scattering. We have used $\vec{p}, \hat{a}^{*}$ to parametrize three on-shell particles, as

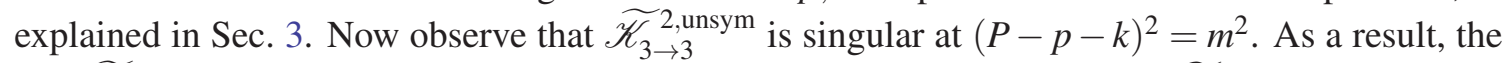
full $\widetilde{\mathscr{K}_{3} \rightarrow 3}$, defined as the sum of all connected three-to-three diagrams with $\widetilde{\mathrm{PV}}$ pole prescription, is singular and does not have a uniformly convergent partial wave expansion. It is therefore difficult to imagine how $\widetilde{\mathscr{K}}_{3} \rightarrow 3$ can be directly extracted from the finite-volume spectrum.

Indeed, it turns out the the quantization condition depends directly not on $\widetilde{\mathscr{K}_{3}} \rightarrow 3$ but instead on a subtracted quantity which is everywhere smooth. Furthermore, the terms that we subtract depend only on on-shell two-to-two K-matrices, which is reasonable since the divergences are due to on-shell intermediate states. This means that one can recover $\widetilde{\mathscr{K}}_{3} \rightarrow 3$ from the spectrum, by first finding the divergence-free object and then adding in the known singular terms. The divergencefree three-to-three K-matrix is defined as

$$
i \widetilde{\mathscr{K}_{\mathrm{df}, 3 \rightarrow 3}}\left(\vec{k}^{\prime}, \hat{a}^{*}, \vec{k}, \hat{a}^{*}\right) \equiv i \widetilde{\mathscr{K}_{3} \rightarrow 3}\left(\vec{k}^{\prime}, \hat{a}^{*}, \vec{k}, \hat{a}^{*}\right)-\mathscr{S}\left[i \mathscr{D}\left(\vec{k}^{\prime}, \hat{a}^{*}, \vec{k}, \hat{a}^{*}\right)\right],
$$

where $\mathscr{S}$ denotes symmetrization of external momenta and $\mathscr{D}$ satisfies an integral equation:

$$
i \mathscr{D}\left(\vec{k}^{\prime}, \hat{a}^{\prime *}, \vec{k}, \hat{a}^{*}\right)=4 \pi Y_{\ell^{\prime}, m^{\prime}}^{*}\left(\hat{a}^{\prime *}\right) i \mathscr{D}_{\ell^{\prime}, m^{\prime} ; \ell, m}\left(\vec{k}^{\prime}, \vec{k}\right) Y_{\ell, m}\left(\hat{a}^{*}\right),
$$




$$
i \mathscr{D}\left(\vec{k}^{\prime}, \vec{k}\right)=i \widetilde{\mathscr{K}}\left(\vec{k}^{\prime}\right) i G_{\infty}\left(\vec{k}^{\prime}, \vec{k}\right) i \widetilde{\mathscr{K}}(\vec{k})+\widetilde{\mathrm{PV}} \int_{\vec{\ell}} \frac{1}{2 \omega_{\ell}} i \widetilde{\mathscr{K}}\left(\vec{k}^{\prime}\right) i G_{\infty}\left(\vec{k}^{\prime}, \vec{\ell}\right) i \mathscr{D}(\vec{\ell}, \vec{k}),
$$

Here $G_{\infty}$ is the three-particle pole transformed to two-particle angular-momentum space, with additional factors included for technical reasons:

$$
G_{\infty ; \ell^{\prime}, m^{\prime}, \ell, m}(\vec{k}, \vec{p}) \equiv \frac{4 \pi Y_{\ell^{\prime}, m^{\prime}}\left(\hat{p}^{*}\right) Y_{\ell, m}^{*}\left(\hat{k}^{*}\right) H(\vec{k}) H(\vec{p})}{2 \omega_{p k}\left(E-\omega_{p}-\omega_{k}-\omega_{p k}\right)}\left(p^{*} / q_{k}^{*}\right)^{\ell^{\prime}}\left(k^{*} / q_{p}^{*}\right)^{\ell} .
$$

In Eq. (5.4), $\mathscr{D}, \widetilde{\mathscr{K}}$ and $G_{\infty}$ are understood to have two sets of implicit angular momentum indices, with internal indices contracted. The starred quantities are defined in Sec. 3.

Solving the integral equation (5.4) iteratively, the result, in schematic form, is $\mathscr{D}=\mathscr{K} G_{\infty} \mathscr{K}+$ $\mathscr{K} G_{\infty} \mathscr{K} G_{\infty} \mathscr{K}+\ldots$. Thus the subtraction in (5.2) removes the infinite sequence of possibly divergent contributions to $\widetilde{\mathscr{K}_{3} \rightarrow 3}$. More precisely, the terms which are divergent are those for which the series of pairwise scatterings is possible classically. In the case of degenerate particles only the first two terms diverge. However, in a generalization to non-degenerate particles, the number of divergent diagrams will depend on the mass ratios. For this reason it is not surprising that our finite-volume analysis leads to a quantity with all terms subtracted.

Since $\widetilde{\mathscr{K}_{\mathrm{df}, 3 \rightarrow 3}}$ is finite for all on-shell momenta, it can be decomposed into spherical harmonics

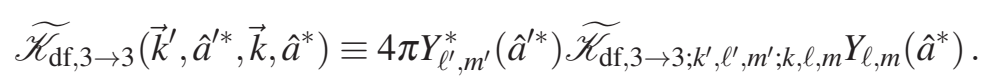

Although $\widetilde{\mathscr{K}}_{\mathrm{df}, 3 \rightarrow 3 ; k^{\prime}, \ell^{\prime}, m^{\prime} ; k, \ell, m}$ is defined for all real $\vec{k}$ and $\vec{p}$, it turns out that our final answer will only depend on the quantity at values $\vec{k}, \vec{p} \in(2 \pi / L) \mathbb{Z}^{3}$. The index notation on the lefthand side of Eq. (5.6) is meant to be suggestive of this discretization. Indeed, from this point on $\widetilde{\mathscr{K}}_{\mathrm{df}, 3 \rightarrow 3 ; k^{\prime}, \ell^{\prime}, m^{\prime} ; k, \ell, m}$ is understood as a discrete matrix in the direct-product space (4.9).

\section{Three-particle quantization condition}

After a lengthy analysis, which we do not describe here, we find the quantization condition ${ }^{2}$

$$
\operatorname{det}\left[1+\widetilde{F}_{3} \widetilde{\mathscr{K}}_{\mathrm{df}, 3 \rightarrow 3}\right]=0
$$

The determinant is over the direct product space (4.9). At fixed $\{L, \vec{P}\}$ the determinant is a function of $E$. The set of solutions $E_{1}, E_{2}, \cdots$ to Eq. (6.1) give the spectrum of the finite-volume theory.

The quantity $\widetilde{F}_{3}$ appearing in Eq. (6.1) depends on the two-to-two K-matrix [packaged into the matrix $\widetilde{\mathscr{K}}$ defined in Eq. (4.10)], as well as the kinematic "sum minus integral" function $\widetilde{F}$ [Eq. (4.11)], and one new kinematic function $G$. Our result is

$$
\widetilde{F}_{3} \equiv \frac{\widetilde{F}}{2 \omega L^{3}}\left[\frac{1}{1+[1+\widetilde{\mathscr{K}} G]^{-1} \widetilde{\mathscr{K} F}}-\frac{2}{3}\right],
$$

\footnotetext{
${ }^{2}$ In the talks, we quoted a final result in which we used the $i \varepsilon$ prescription for infinite volume quantities rather than our $\widetilde{\mathrm{PV}}$ prescription. The form of the result was the same as Eq. (6.1), but had $\widetilde{\mathscr{M}}_{\mathrm{df}, 3 \rightarrow 3}$ (the divergence-free scattering amplitude) in place of $\widetilde{\mathscr{K}}_{\mathrm{df}, 3 \rightarrow 3}$, and the definition of $\widetilde{F}_{3}$ was also slightly changed. This result ignored the impact of cusp singularities and was incorrect (since not all power-law volume effects were included).
} 
All quantities are matrices with pairs of implicit $k, l, m$ indices. The function $G$ is simply $G_{\infty}$ [Eq. (5.5)] evaluated for discrete momenta, aside from a simple change in overall normalization:

$$
G_{k, \ell^{\prime}, m^{\prime} ; p, \ell, m} \equiv \frac{1}{2 \omega_{p} L^{3}} \frac{4 \pi Y_{\ell^{\prime}, m^{\prime}}\left(\hat{p}^{*}\right) Y_{\ell, m}^{*}\left(\hat{k}^{*}\right) H(\vec{k}) H(\vec{p})}{2 \omega_{p k}\left(E-\omega_{p}-\omega_{k}-\omega_{p k}\right)}\left(p^{*} / q_{k}^{*}\right)^{\ell^{\prime}}\left(k^{*} / q_{p}^{*}\right)^{\ell} .
$$

We now give a brief discussion of the result (6.1). We first note the superficial similarity to the two-particle quantization condition, already given in two forms above [Eqs. (4.12) and (4.13)], and which can also be written

$$
\operatorname{det}\left[1+\widetilde{F_{2}} \widetilde{\mathscr{K}_{2}}\right]=0
$$

This form provides a clear separation between infinite-volume quantities related to scattering (here the two-to-two K-matrix) and finite-volume effects entering through the kinematical function $\widetilde{F}_{2}$. It shows that the spectrum depends only on the infinite-volume scattering amplitude [related to the K-matrix through Eq. (4.14)].

The same comments hold for our three-particle result, Eq. (6.1), though with some subtleties. First, $\widetilde{F}_{3}$ is not simply a kinematical function, but rather a kinematical function $(\widetilde{F}$-which is simply $\widetilde{F}_{2}$ multiplied by $\delta_{k, k^{\prime}}$ ) decorated by contributions from two-to-two scattering. One can see from Eq. (6.2) that this decoration consists of two nested geometric series, one involving the two-particle K-matrix, and the other the "switch-factor" $G$. These arise, respectively from diagrams in the second and third/fourth lines of Fig. 1. Such "decoration" seems an unavoidable consequence of moving from two to three particles.

The second subtlety concerns the appearance of sub-threshold two-to-two amplitudes. This point was first noted and discussed in Ref. [4]. They arise in $\widetilde{\mathscr{K}}$ when the spectator momentum is such that the remaining two particles are below threshold. Such amplitudes can be defined by analytically continuing the two-particle K-matrix from the physical region, as is, in fact, routinely done when using the two-particle quantization condition to discuss bound states. The regulator function $H$ in our pole prescription ensures that we only need to analytically continue for a distance $\sim m$.

The final subtlety concerns the connection with infinite-volume quantities. Our result shows that the spectrum is determined by infinite-volume quantities related to scattering, as was previously found in the non-relativistic treatment of Ref. [4]. However, the quantities which appear are not the scattering amplitudes themselves, but differ in two ways. First, our result contains divergence-free three-particle quantities. Second, our quantities are defined using the $\widetilde{P V}$, rather than the $i \varepsilon$, pole prescription. Thus they are a version of K-matrices having some regulator dependence entering through the choice of the function $H$. We do not think that either difference is a serious concern. The full amplitude can be reconstructed from the divergence-free one using the integral equation (5.4). Similarly, we can write an integral equation relating $\widetilde{\mathscr{K}}_{\mathrm{df}, 3 \rightarrow 3}$ to $\widetilde{\mathscr{M}}_{\mathrm{df}, 3 \rightarrow 3}$. These equations can be solved numerically once the approximations to the scattering amplitudes discussed below are made. Thus, our intermediate quantity $\widetilde{\mathscr{K}}_{\mathrm{df}, 3 \rightarrow 3}$ is directly related to $\mathscr{M}_{3 \rightarrow 3}$. Furthermore, it is arguable that $\widetilde{\mathscr{K}}_{\mathrm{d}, 3 \rightarrow 3}$ is a more natural quantity to appear than $\mathscr{M}_{3 \rightarrow 3}$, since it is a smooth, real function (without cusps or singularities) and thus is simpler to approximate. 


\section{Truncation}

Our quantization condition (6.1) is a formal equation involving infinite-dimensional matrices. The same is true of the two-particle quantization condition (6.4) [or equivalently Eq. (4.12)]. To make these equations useful in practice one must develop systematic approximation schemes which truncate the matrices down to finite dimensions.

In the two-particle case, this is justified because of the rapid decrease of elastic scattering amplitudes with increasing $\ell$ at fixed energy. Mathematically, one must assume that $\mathscr{M}_{2}$ vanishes for $\ell>\ell_{\max }$ (which implies that $\widetilde{\mathscr{K}_{2}}$ vanishes also). Then, even though $\widetilde{F}_{2}$ is not diagonal, one can show that the determinant truncates to that of a $\left(2 \ell_{\max }+1\right)^{2}$ matrix (with further simplifications due to residual symmetries whose details depend on $\vec{P}$ ). The simplest case is $\ell_{\max }=0$, and this is often the form used in practice. In any case, the key point is that truncation leads to an algebraic equation involving $\widetilde{\mathscr{K}_{2}}$ evaluated at the CM energy $E^{*}=\sqrt{E^{2}-\vec{P}^{2}}$ (with $E$ the energy of the spectral line).

What happens in the $3 \rightarrow 3$ case? Here we have a larger index space, involving the spectator momentum $\vec{k}$. The key observation, however, is that the sum over $\vec{k}$ is self-truncating. To understand this, note that the four-momentum flowing into the non-spectator pair is $p_{2}=\left(E-\omega_{k}, \vec{P}-\vec{k}\right)$. For fixed $(E, \vec{P})$, as $|\vec{k}|$ increases, $p_{2}^{2}$ decreases below the threshold value $4 m^{2}$. In this subthreshold region (for the non-spectator pair) the intermediate 3-particle state cannot go on shell, and the sum-integral difference $\widetilde{F}$ [Eq. (4.11)] becomes exponentially suppressed. Although formally true immediately below threshold, $p_{2}^{2}$ must be reduced by $\sim m^{2}$ below the threshold value $4 m^{2}$, before the exponential suppression becomes numerically significant. This is because, roughly speaking, the exponent is $L\left(4 m^{2}-p_{2}^{2}\right) / m$. This is why one must allow a range of subthreshold momenta to have an accurate quantization condition.

Our definition of $\widetilde{F}$ includes, in addition, the regulator function $H(\vec{k})$, which smoothly sets $\widetilde{F}$ to zero as $p_{2}^{2}$ approaches 0 (which is well into the regime where $\widetilde{F}$ is exponentially suppressed). Thus we have an exact truncation, at the cost of an exponentially small error.

The upshot is that the $\vec{k}$ sum is truncated, with say $N$ terms to be kept. One then assumes that $\widetilde{\mathscr{K}}_{2}$ can be truncated at $\ell_{\max , 2}$, as in the two-particle case, and that $\widetilde{\mathscr{K}}_{\mathrm{df}, 3 \rightarrow 3}$ can be truncated at $\ell_{\text {max }, 3}$. Here the prior removal of the singular part of $\widetilde{\mathscr{K}_{\mathrm{df}}, 3 \rightarrow 3}$ is key, since otherwise truncation is not justified. Calling $\ell_{\max }$ the larger of $\ell_{\max , 2}$ and $\ell_{\max , 3}$, one can then show that the determinant equation collapses to one for matrices of dimension $\left[N\left(2 \ell_{\max }+1\right)\right]^{2}$.

The end result is an algebraic equation involving the various non-vanishing partial waves of $\widetilde{\mathscr{K}_{2}}$ and $\widetilde{\mathscr{K}}_{\mathrm{df}, 3 \rightarrow 3}$, the latter evaluated at the $\mathrm{CM}$ energy $E^{*}$ of the spectral line. Assuming that $\mathscr{M}_{2}$ is known from studies of two-particle spectra using the Lüscher method, and interpolating as necessary, one gains information about $\widetilde{\mathscr{K}_{\mathrm{df}}, 3 \rightarrow 3}$ from each spectral line. To make progress, one would likely need to parametrize $\widetilde{\mathscr{K}}_{\mathrm{df}, 3 \rightarrow 3}$ as a function of $\vec{k}, \ell$ and $m$, and then use as many spectral lines as parameters to determine the latter (e.g. by varying $\vec{P}$, but keeping $E^{*}$ fixed).

\section{Threshold expansion}

An important check on our formalism is provided by the threshold expansion. This applies at $\vec{P}=0$, where the lightest non-interacting 3-particle state has $E=3 m$ (with each particle at rest). Interactions shift this by $\delta E \propto 1 / L^{3}$. For large $L, \delta E \ll m$, and the particles are non-relativistic. 
In addition, our analysis shows that the relativistic transitions $(3 \rightarrow 1,3 \rightarrow 5$, etc.) do not play an essential role, leading to non-analyticities only at energies beyond the range we consider. Thus one can calculate $\delta E$ in this limit using a non-relativistic theory. Such a calculation has been carried out in Refs. [9, 10], with $\delta E$ obtained through ${ }^{3} \mathscr{O}\left(1 / L^{6}\right)$. Our formalism should reproduce this result.

Close to threshold one can truncate the two-particle interactions to be purely s-wave $\left(\ell_{\max , 2}=\right.$ 0 ) and take $\widetilde{\mathscr{K}_{\mathrm{df}, 3 \rightarrow 3}}$ to be a constant (implying $\ell_{\max , 3}=0$ too). ${ }^{4}$ Thus $\ell_{\max }=0$ and the only matrix structure is in spectator-momentum space. Furthermore, one can show that the dominant term in $\left[\widetilde{F}_{3}\right]_{\vec{k}, \vec{k}^{\prime}}$ is that with $\vec{k}=\vec{k}^{\prime}=0$, other matrix elements being suppressed by powers of $1 / L$. Indeed we will see that, to the order we are working, only the $\vec{k}=\vec{k}^{\prime}=0$ entry needs to be included. This means that the quantization condition (6.1) collapses to

$$
\left[\widetilde{F}_{3}\right]_{\overrightarrow{0}, \overrightarrow{0}} \widetilde{\mathscr{K}_{\mathrm{df}, 3 \rightarrow 3}}=-1
$$

It is now useful to rewrite $\widetilde{F}_{3}$ as

$$
\widetilde{F}_{3}=\frac{\widetilde{F}}{2 \omega L^{3}}\left[\frac{1}{3}+\frac{1}{\widetilde{\mathscr{K}}_{2}^{-1}+\widetilde{F}+G} \widetilde{F}\right]
$$

From (8.1) we know that $\left[\widetilde{F}_{3}\right]_{\overrightarrow{0}, \overrightarrow{0}}$ scales as $L^{0}$ (since $\widetilde{\mathscr{K}}_{\mathrm{df}, 3 \rightarrow 3}$ is a constant). However, $\widetilde{F}_{3}$ contains an explicit factor of $L^{-3}$. One can show that $\widetilde{F} \sim L^{0}$ near threshold, so the only way that the overall $L^{-3}$ factor can be canceled is if $\left(\widetilde{\mathscr{K}_{2}^{-1}}+\widetilde{F}+G\right) \sim L^{-3}$. Thus the $L^{0}, L^{-1}$ and $L^{-2}$ parts of this combination must be canceled by tuning $\delta E$. This turns out to determine the $L^{-3}, L^{-4}$ and $L^{-5}$ parts of $\delta E$, respectively. The $L^{-6}$ part is then determined by enforcing Eq. (8.1). We stress that the full matrix inverse of $\left(\widetilde{\mathscr{K}}_{2}^{-1}+\widetilde{F}+G\right)$ must be evaluated (to the order we are working), even though the external indices are fixed to $\overrightarrow{0}$.

After a moderately lengthy calculation we find

$$
\begin{aligned}
\delta E= & \frac{12 \pi a}{m L^{3}}\left[1-\frac{a}{\pi L} I+\left(\frac{a}{\pi L}\right)^{2}\left(I^{2}+J\right)+\left(\frac{a}{\pi L}\right)^{3}\left(-I^{3}+I J+15 K-16 Q-8 R\right)\right] \\
& +\frac{72 a^{3} \pi^{2} r}{m L^{6}}+\frac{36 a^{2} \pi^{2}}{m^{3} L^{6}}-\frac{\widetilde{\mathscr{K}_{\mathrm{df}} 3 \rightarrow 3}}{48 m^{3} L^{6}}+\mathscr{O}\left(L^{-7}\right) .
\end{aligned}
$$

Here $a$ is the 2-particle scattering length (with the nuclear physics sign convention: positive for repulsion), and $r$ is the corresponding effective range. $I, J$ and $K$ are familiar finite-volume zeta functions involving sums over integer vectors:

$$
I=\sum_{\vec{n} \neq 0}^{|\vec{n}|=\Lambda} \frac{1}{\vec{n}^{2}}-4 \pi \Lambda, \quad J=\sum_{\vec{n} \neq 0} \frac{1}{\left(\vec{n}^{2}\right)^{2}}, \quad K=\sum_{\vec{n} \neq 0} \frac{1}{\left(\vec{n}^{2}\right)^{3}} .
$$

\footnotetext{
${ }^{3}$ Subsequent work has pushed this to one higher order, but we will not need this result.

${ }^{4}$ At threshold $\widetilde{\mathscr{K}}_{\mathrm{df}, 3 \rightarrow 3}$ and $\mathscr{M}_{\mathrm{df}, 3 \rightarrow 3}$ are equal. Thus the results in this section are unaffected by the presence of cusps. It turns out that they enter the threshold expansion first at $\mathscr{O}\left(1 / L^{10}\right)$.
} 
They have known numerical values. $Q$ and $R$ are more complicated quantities:

$$
\begin{gathered}
Q=-2048 \pi^{6} L^{3} m^{3} \sum_{\vec{k} \neq 0, \vec{p} \neq 0} G_{\overrightarrow{0}, \vec{k}} G_{\vec{k}, \vec{p}} G_{\vec{p}, \overrightarrow{0}}=\sum_{\vec{n}_{k} \neq 0, \vec{n}_{p} \neq 0}^{\mathrm{reg}} \frac{1}{\vec{n}_{k}^{2} \vec{n}_{p}^{2}\left[\vec{n}_{k}^{2}+\vec{n}_{p}^{2}+\left(\vec{n}_{k}+\vec{n}_{p}\right)^{2}\right]}+\mathscr{O}\left(L^{-1}\right) \\
R=-4096 \pi^{6} L^{3} m^{3} \sum_{\vec{k} \neq 0} G_{\overrightarrow{0}, \vec{k}} \widetilde{F}_{\vec{k}, \vec{k}} G_{\vec{k}, \overrightarrow{0}}=\sum_{\vec{n}_{k} \neq 0} \frac{1}{\left(\vec{n}_{k}^{2}\right)^{2}} \sum_{\vec{n}_{p}}^{\text {reg }} \frac{1}{\vec{n}_{k}^{2}+\vec{n}_{p}^{2}+\left(\vec{n}_{k}+\vec{n}_{p}\right)^{2}}+\mathscr{O}\left(L^{-1}\right)
\end{gathered}
$$

where $\vec{k}=2 \pi \vec{n}_{k} / L$ and $\vec{p}=2 \pi \vec{n}_{p} / L$. For both $Q$ and $R$, the first form of the result shows how the matrix nature (in spectator momentum space) of $G$ and $\widetilde{F}$ enters. In addition, both of these forms are convergent sums. When expanded in the non-relativistic limit, however, one ends up with divergent sums (the second expressions) which must be regulated. It is the latter forms that appear in the results of Refs. [9, 10].

The first line of our result for $\delta E$, Eq. (8.3), agrees with the results of Refs. [9, 10]. Various aspects of this agreement are noteworthy. The leading and first sub-leading terms $\left(L^{-3}\right.$ and $L^{-4}$, respectively) are simply the result for two-particles multiplied by a factor of 3 . This corresponds to the presence of three possible two-particle pairs. At $\mathscr{O}\left(L^{-5}\right)$, however, features enter which are special to the 3-particle case (leading to a flip in the sign of the $J$ term). In addition, the switch factors $G$ play an essential role in our calculation, and without their presence we would not find agreement with Refs. [9, 10].

We turn now to the remaining $L^{-6}$ terms on the second line of (8.3). Here the situation is more complicated. In the first of these terms, we have a factor of 72, while Ref. [9] finds 24 and Ref. [10] has 36. The second of our terms (that proportional to $a^{2}$ ) is absent in Refs. $[9,10]$. And in the third of the terms, our K-matrix $\widetilde{\mathscr{K}_{\mathrm{df}, 3 \rightarrow 3}}$ is replaced by an unphysical, regulator-dependent quantity. For example, Ref. [9] has $\eta_{3}(\mu) / L^{6}$, with $\eta_{3}$ a contact 3-particle potential and $\mu$ a renormalization scale. Finally, Refs. $[9,10]$ each have an additional regulator-dependent term, e.g.

$$
\frac{64 \pi a^{4}}{m L^{6}}(3 \sqrt{3}-4 \pi) \log (\mu L)
$$

in Ref. [9]. This term arises when dimensional regularization is used to define the second forms in Eqs. $(8.5,8.6)$.

We stress that, despite the discrepancies in the form of $L^{-6}$ terms, there is no conflict between our result and those of Refs. [9, 10]. We do not, a priori, know the relation between $\widetilde{\mathscr{K}}_{\mathrm{df}, 3 \rightarrow 3}$ and $\eta_{3}(\mu)$. One is a physical quantity, while the other is a short-distance unphysical parameter, as evidenced by its regulator dependence. It follows the terms on the second line of (8.3) do not provide a consistency check, but instead imply a relation between three-body parameters that is required for the energy shifts to match. As far as we can see, there is nothing forbidding this relation to include the finite $a^{2}$ and $a^{2} r$ terms. Indeed, a similar finite difference is required to match the results of Refs. [9] and [10]. Nevertheless, it would clearly be good to check this purported relation in another context.

\section{Outlook}

Having a formalism is only the first step. We are presently investigating the practicality of the (truncated) quantization condition using simple models for the scattering amplitudes. It is also 
important to understand more clearly the relationship of our approach to those of Refs. $[4,5]$ as well as to that of HAL-QCD [11].

We also plan to extend the theoretical work in several directions: working with non-identical non-degenerate particles, generalizing the Lellouch-Lüscher relation to three particles, and extending our result to cases in which the two-particle K-matrices can have poles above threshold, i.e. in which there are two-particle resonances within the kinematic range of our formula. Ultimately, we aim to consider the four-particle case.

\section{Acknowledgments}

We thank Raul Briceno, Zohreh Davoudi and Akaki Rusetsky for helpful discussions. MTH was supported in part by a Fermilab Fellowship in Theoretical Physics. Fermilab is operated by Fermi Research Alliance, LLC, under Contract No. DE-AC02-07CH11359 with the United States Department of Energy (DOE). MTH and SRS were supported in part by DOE grant No. DE-FG0296ER40956.

\section{References}

[1] M. Lüscher, Commun. Math. Phys. 104, 177 (1986).

[2] M. Lüscher, Commun. Math. Phys. 105, 153 (1986).

[3] M. Lüscher, Nucl. Phys. B354, 531 (1991).

[4] K. Polejaeva and A. Rusetsky, Eur.Phys.J. A48, 67 (2012), arXiv:1203.1241 [hep-lat] .

[5] R. A. Briceno and Z. Davoudi, Phys.Rev. D87, 094507 (2013), arXiv:1212.3398 [hep-lat] .

[6] M. T. Hansen and S. R. Sharpe, in preparation .

[7] C. Kim, C. Sachrajda, and S. R. Sharpe, Nucl.Phys. B727, 218 (2005), arXiv:hep-lat/0507006 [hep-lat] .

[8] K. Rummukainen and S. A. Gottlieb, Nucl.Phys. B450, 397 (1995), arXiv:hep-lat/9503028 [hep-lat] .

[9] S. R. Beane, W. Detmold, and M. J. Savage, Phys.Rev. D76, 074507 (2007), arXiv:0707.1670 [hep-lat] .

[10] S. Tan, Phys.Rev. A78, 013636 (2008), arXiv:0709.2530 [cond-mat.stat-mech] .

[11] S. Aoki, N. Ishii, T. Doi, Y. Ikeda, and T. Inoue, Phys.Rev. D88, 014036 (2013), arXiv:1303.2210 [hep-lat] . 